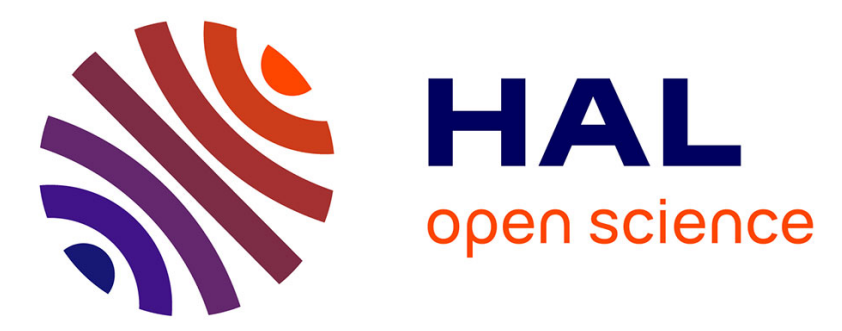

\title{
Object Association with Belief Functions, an application with vehicles
}

\author{
David Mercier, Eric Lefevre, Daniel Jolly
}

\section{To cite this version:}

David Mercier, Eric Lefevre, Daniel Jolly. Object Association with Belief Functions, an application with vehicles. Information Sciences, 2011, 181 (24), pp.5485-5500. 10.1016/j.ins.2011.07.045 . hal03354177

\section{HAL Id: hal-03354177 \\ https://hal.science/hal-03354177}

Submitted on 24 Sep 2021

HAL is a multi-disciplinary open access archive for the deposit and dissemination of scientific research documents, whether they are published or not. The documents may come from teaching and research institutions in France or abroad, or from public or private research centers.
L'archive ouverte pluridisciplinaire HAL, est destinée au dépôt et à la diffusion de documents scientifiques de niveau recherche, publiés ou non, émanant des établissements d'enseignement et de recherche français ou étrangers, des laboratoires publics ou privés. 


\title{
Object Association with Belief Functions, an application with vehicles.
}

\author{
David Mercier, Éric Lefèvre, Daniel Jolly \\ Univ. Lille Nord de France, UArtois, EA 3926 LGI2A, France
}

\begin{abstract}
The problem tackled in this article consists in associating perceived objects detected a certain time with known objects previously detected, knowing uncertain and imprecise information regarding the association of each perceived objects with each known objects. For instance, this problem can occur during the association step of an association obstacle tracking process, especially in the context of vehicle driving aid. A contribution in the modelling of this association problem in the belief function framework is introduced. By interpreting belief functions as weighted opinions according to the Transferable Belief Model semantics, pieces of information regarding the association of known objects and perceived objects can be expressed in a common global space of association to be combined by the conjunctive rule of combination, and a decision making process using the pignistic transformation can be made. This approach is validated on real data.
\end{abstract}

Keywords: Object association, obstacle tracking, belief functions, Transferable Belief Model.

\section{Introduction}

In obstacle tracking, the association step consists in establishing a correlation between tracks (known objects) and targets (perceived objects) from information usually provided by different sensors or captors. Such a mapping can be even more complex depending on the number of targets and tracks, as well as the quality of the provided information. Introduced by Dempster [7] and Shafer [26], belief functions constitute a suitable framework for the representation and manipulation of imperfect information [4, 12, 13, 20, 30, 31]. Thus, next to architectures based on Bayesian probabilistic framework [2, 3], Rombaut $[23,24]$ develops a first modelling based on belief functions. In this 
model, information regarding the association of couples (known objects, perceived objects) is represented by belief functions, which are combined using, for simplicity reasons, an adapted combination introduced by Rombaut. In [16] this latter model is developed by using a decision-making system based on belief matrices and the application of a coupling algorithm.

In this article, a modelling of this association step problem is introduced in the Smets' semantic approach of belief functions: the Transferable Belief Model (TBM) [29], a subjectivist and non-probabilistic interpretation of the Dempster-Shafer theory of belief function. In particular, it is shown that TBM classical tool like the conjunctive combination rule and the pignistic decision-making can be implemented and tested in a real time application, these experimental results demonstrating the effectiveness of this approach as compared to Rombaut's combination rule.

The works presented here extend a short version of this study presented in [19], as well as it reexpress and extend in the Transferable Belief Model a former model presented by some of the authors in [17]. At last, the association problem described here also presents some similarities with the works undertaken by Ristic and Smets in [22], which are discussed in this article.

This article is organized as follows. The TBM basic concepts we need are recalled in Section 2. An association algorithm based on belief functions is then introduced in Section 3 and discussed with previous approaches in Section 4. Then, experimental results on real data are presented in Section 5. Finally, Section 6 concludes this article.

\section{Transferable Belief Model (TBM): basic concepts}

The Transferable Belief Model (TBM) is a model of uncertain reasoning and decision-making based on two levels [14, 29]:

- the credal level, where available pieces of information are represented by belief functions, and manipulated;

- the pignistic or decision level, where belief functions are transformed into probability measures when a decision has to be made, and the expected utility is maximized.

\subsection{Representing information with belief functions}

\subsubsection{Belief functions}

The knowledge held by an agent is represented by the allocation of a finite mass of belief to subsets of the universe of discourse. 
Let $\Omega=\left\{\omega_{1}, \omega_{2}, \ldots, \omega_{N}\right\}$, called the frame of discernment, be a finite set composed of all possible answers to a given question $Q$ of interest. The beliefs held by a rational agent $A g$ regarding the answer to question $Q$ can be quantified by a mass function or a basic belief assignment (BBA) $m_{A g}^{\Omega}: 2^{\Omega} \rightarrow[0,1]$ s.t.:

$$
\sum_{A \subseteq \Omega} m_{A g}^{\Omega}(A)=1
$$

The quantity $m_{A g}^{\Omega}(A)$ represents the part of the unit mass allocated to the hypothesis that the answer to question $Q$ is in the subset $A$ of $\Omega$. When there is no ambiguity, the notation $m_{A g}^{\Omega}$ will be simplified as follows $m^{\Omega}$ or $m$.

- A subset $A$ of $\Omega$ such that $m(A)>0$ is called a focal set of $m$.

- A BBA $m$ with only one focal set $\mathrm{A}$ is called a categorical $B B A$ and is denoted $m_{A}$; then $m_{A}(A)=1$.

- Total ignorance is represented by the BBA $m_{\Omega}$ called the vacuous $B B A$.

- A normal $B B A m$ satisfies the condition $m(\emptyset)=0$.

- Let $A$ be a subset of $\Omega$, the cardinality of $A$, denoted $|A|$, is the number of elements of $\Omega$ in $A$; if $|A|=1, A$ is said to be a singleton.

The belief and plausibility functions associated with a BBA $m$ are defined, respectively, as:

$$
\begin{aligned}
\operatorname{bel}(A)=\sum_{\emptyset \neq B \subseteq A} m(B) & \forall A \subseteq \Omega, \\
\operatorname{pl}(A)=\sum_{B \cap A \neq \emptyset} m(B) & \forall A \subseteq \Omega .
\end{aligned}
$$

Functions $m$, bel and $p l$ are in one-to-one correspondence, and thus constitute different forms of the same information.

\subsubsection{Refinements and Coarsenings}

When applying the TBM to a real-world application, the determination of the frame of discernment $\Omega$, which defines the set of states on which beliefs will be expressed, is a crucial step. As noticed by Shafer [26, chapter $6]$, the degree of granularity of $\Omega$ is always, to some extent, a matter of 
convention, as any element of $\Omega$ representing a given state can always be split into several alternatives. Hence, it is fundamental to examine how a belief function defined on a frame may be expressed in a finer or, conversely, in a coarser frame. The concepts of refinement and coarsening can be defined as follows.

Let $\Theta$ and $\Omega$ denote two frames of discernment. A mapping $\rho: 2^{\Theta} \rightarrow 2^{\Omega}$ is called a refining of $\Theta$ (Figure 5 ) if it verifies the following properties:

1. The set $\{\rho(\{\theta\}), \theta \in \Theta\} \subseteq 2^{\Omega}$ is a partition of $\Omega$, and

2. For all $A \subseteq \Theta$ :

$$
\rho(A)=\bigcup_{\theta \in A} \rho(\{\theta\}) .
$$

$\Theta$ is then called a coarsening of $\Omega$, and $\Omega$ is called a refinement of $\Theta$.

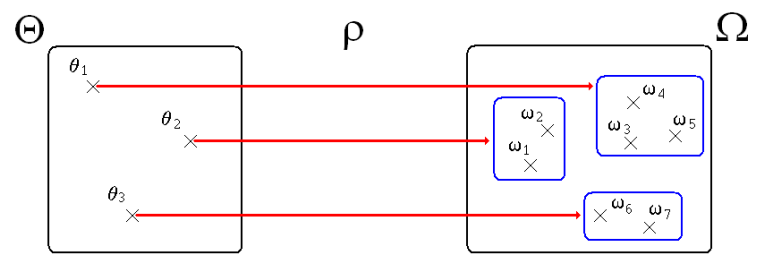

Figure 1: Illustration of a coarsening $\Theta$ of $\Omega$ associated with a refining $\rho$ of $\Theta$.

\subsection{Manipulating information with belief functions}

\subsubsection{Vacuous extension}

The vacuous extension operation allows one to convey a mass function $m^{\Theta}$, expressing a state of belief on $\Theta$, to a finer frame $\Omega$, a refinement of $\Theta$. Stemming from the least committed principle [27], this operation is denoted with an arrow pointing up, and is defined by:

$$
m^{\Theta \uparrow \Omega}(\rho(A))=m^{\Theta}(A), \quad \forall A \subseteq \Theta,
$$

where $\rho$ is the refining of $\Theta$ in $\Omega$.

\subsubsection{Combining beliefs}

Two BBAs $m_{1}$ and $m_{2}$, induced by distinct and reliable sources of information, can be combined using the conjunctive rule of combination (CRC), also called unnormalized Dempster's rule of combination, defined for all $A \subseteq \Omega$ by:

$$
m_{1} @ m_{2}(A)=\sum_{B \cap C=A} m_{1}(B) m_{2}(C) .
$$


The normalization hypothesis $(m(\emptyset)=0)$ can be recovered with the following normalization step:

$$
m_{1} \oplus m_{2}(A)= \begin{cases}\frac{m_{1} @_{2}(A)}{1-m_{1} @ 2(\emptyset)} & \text { if } \emptyset \neq A \subseteq \Omega, \\ 0 & \text { otherwise. }\end{cases}
$$

This latter rule of combination is called Dempster's rule of combination.

\subsection{Decision-making level}

When a decision has to be made regarding the answer to question $\mathrm{Q}$, some rationally principles [6] justify the strategy consisting in choosing the decision $d$ among a set of possible decisions $\mathcal{D}$, which minimizes the expected risk defined by:

$$
R(d)=\sum_{\omega \in \Omega} c(d, \omega) P^{\Omega}(\{\omega\}),
$$

where $P^{\Omega}: 2^{\Omega} \rightarrow[0,1]$ is a probability measure and $c: \mathcal{D} \times \Omega \rightarrow \mathbb{R}$ a cost function, $c(d, \omega)$ representing the cost to decide $d$ while the truth is $\omega$.

At this level, the mass function $m^{\Omega}$ representing the available information regarding the answer to question $\mathrm{Q}$ belonging to $\Omega$ (resulting in practice from a fusion process) has then to be transformed in a probability measure. A solution [9] consists in computing the pignistic probability [28] defined by:

$$
\operatorname{Bet}^{\Omega}(\{\omega\})=\sum_{\{A \subseteq \Omega, \omega \in A\}} \frac{m(A)}{|A|(1-m(\emptyset))}, \quad \forall \omega \in \Omega .
$$

The chosen decision is then the one that minimizes the pignistic risk defined by:

$$
R_{B e t}(d)=\sum_{\omega \in \Omega} c(d, \omega) B e t P^{\Omega}(\{\omega\}) .
$$

In the case of $0-1$ costs with $\mathcal{D}=\Omega$, which means that $c\left(\omega_{i}, \omega_{j}\right)=1$ if $i=j, 0$ otherwise, choosing the decision $d$ which minimises the pignistic risk (10) is equivalent to choose the decision $d$ which maximizes the pignistic probability (9).

An other case consists in choosing 0-1 costs with $\mathcal{D}=\Omega \cup\left\{d_{0}\right\}$, where $d_{0}$, called rejection decision [9], consists in refusing to make a decision belonging to $\mathcal{D} \backslash\left\{d_{0}\right\}$ when the risk is judged too high. By denoting $c_{0}=c\left(d_{0}, \omega_{i}\right) \forall i \in$ $\{1, \ldots, N\}$, minimizing the pignistic risk (10) is equivalent to choose the decision:

- $d_{0}$ if $\max _{i=1, \ldots, N} \operatorname{Bet} P\left(\left\{\omega_{i}\right\}\right)<1-c_{0}$, 
- $\omega_{j}$ if $\operatorname{Bet} P\left(\left\{\omega_{j}\right\}\right)=\max _{i=1, \ldots, N} \operatorname{Bet} P\left(\left\{\omega_{i}\right\}\right) \geq 1-c_{0}$.

The cost $c_{0}$ is called the rejection cost.

\section{Object association algorithm}

\subsection{Representing information with belief functions}

The first step when building belief functions is to define the universe of discourse.

Let us consider the following notations:

- $X_{i}$ : represents a perceived object at time $t, i \in I=\{1, \ldots, N\}, N$ being the number of perceived objects at time $t$;

- $Y_{j}$ : represents a known object at previous time $t-1, j \in J=$ $\{1, \ldots, M\}, M$ being the number of known objects at time $t-1$;

- *: is a proposition meaning "no object".

The association process objective consists in finding the best possible association between a set of perceived objects $\left\{X_{1}, X_{2}, \ldots, X_{N},{ }^{\star}\right\}$ and a set of known objects $\left\{Y_{1}, Y_{2}, \ldots, Y_{M},{ }^{\star}\right\}$, under the following constraints:

- each perceived object $X_{i}$ is associated with at most one known object;

- each known object $Y_{j}$ is associated with at most one perceived object;

- proposition * can be associated with any objects.

The frames of discernment involved in this application are then the followings:

- $\Omega_{i, j}=\left\{y_{i, j}, n_{i, j}\right\}$ : contains the two possible answers (yes or no) to the question $Q_{i, j}$ : "Is the perceived object $X_{i}$ associated with the known object $Y_{j}$ ?";

- $\Omega_{X_{i}}=\left\{Y_{1}, Y_{2}, \ldots, Y_{M},{ }^{\star}\right\}$ : contains the set of possible answers to the question $Q_{X_{i}}$ : "Who is associated with the perceived object $X_{i}$ ?", proposition * meaning that $X_{i}$ has appeared;

- $\Omega_{Y_{j}}=\left\{X_{1}, X_{2}, \ldots, X_{N},{ }^{\star}\right\}$ : contains the set of possible answers to the question $Q_{Y_{j}}$ : "Who is associated with the known object $Y_{j}$ ?", proposition * meaning that $Y_{j}$ has disappeared or is hidden. 
Let us remark that $\Omega_{Y_{j}}=\Omega_{Y_{k}}$, for all $j, k \in J$, and $\Omega_{X_{i}}=\Omega_{X_{\ell}}$, for all $i, \ell \in I$. Thus, $\Omega_{X_{i}}$ (respectively $\Omega_{Y_{j}}$ ) can be denoted $\Omega_{X} \forall i$ (respectively $\Omega_{Y} \forall j$ ). At last, when there is no ambiguity, the frames elements will be simplified as follows:

- $\Omega_{X_{i}}=J \cup\{\star\}=\{1, \ldots, M, \star\}$,

- $\Omega_{Y_{j}}=I \cup\{\star\}=\{1, \ldots, N, \star\}$.

In the domain of intelligent vehicles, sensors or measures generally provide information regarding the association between each perceived object $X_{i}$ and each known object $Y_{j}[23,24,16,15]$. More precisely, initial information is represented by mass functions $m^{\Omega_{i, j}}$ on frames $\Omega_{i, j}, i \in I, j \in J$ :

- the mass allocated to $\left\{y_{i, j}\right\}$ expresses information on the fact that $X_{i}$ is associated with $Y_{j}$;

- the mass allocated to $\left\{n_{i, j}\right\}$ expresses information on the fact that $X_{i}$ is not associated with $Y_{j}$;

- the mass allocated to $\Omega_{i, j}=\left\{y_{i, j}, n_{i, j}\right\}$ expresses the ignorance regarding the association of $X_{i}$ and $Y_{j}$.

The association problem to solve can then be express in the following manner: $N \times M$ mass functions $m^{\Omega_{i, j}}$ being defined regarding the association of each perceived object $X_{i}$ with each known object $Y_{j}$, how these pieces of information can be fused/merged to determine:

- Where do perceived objects $X_{i}$ come from?

- What are known objects $Y_{j}$ become?

\subsection{Expressing pieces of information in a common frame}

To answer these questions, the $N \times M$ mass functions can be combined when expressed on two possible common frames: $\Omega_{X}$ and $\Omega_{Y}$.

Frames $\Omega_{X_{i}}$ and $\Omega_{Y_{j}}$ being refinements of $\Omega_{i, j}$, each information $m^{\Omega_{i, j}}$ can be expressed either on $\Omega_{X_{i}}$ or on $\Omega_{Y_{j}}$ by a vacuous extension (5):

$$
m^{\Omega_{i, j} \uparrow \Omega_{X_{i}}}\left(\rho_{i, j}(A)\right)=m^{\Omega_{i, j}}(A), \quad \forall A \subseteq \Omega_{i, j},
$$

where $\rho_{i, j}$ is the refining of $\Omega_{i, j}$ on $\Omega_{X_{i}}$ illustrated in Figure 2, and defined by $\rho_{i, j}\left(\left\{y_{i, j}\right\}\right)=\{j\}$ and $\rho_{i, j}\left(\left\{n_{i, j}\right\}\right)=\overline{\{j\}}$. 


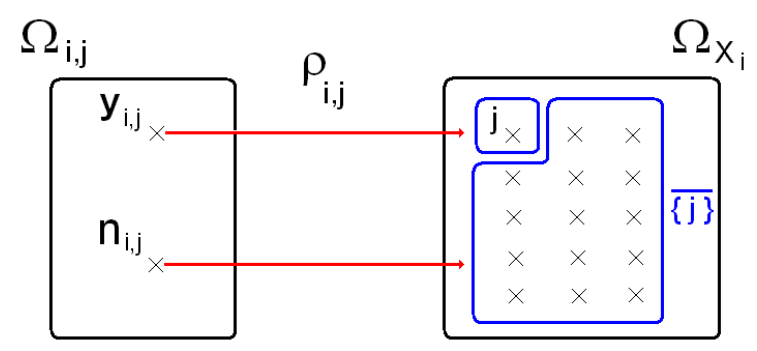

Figure 2: Refining $\rho_{i, j}$ allowing one to transport the information $m^{\Omega_{i, j}}$ on $\Omega_{X_{i}}$.

Thus, for all $(i, j) \in I \times J$ :

$$
\begin{cases}m^{\Omega_{i, j} \uparrow \Omega_{X_{i}}}(\{j\}) & =m^{\Omega_{i, j}}\left(\left\{y_{i, j}\right\}\right) \\ m^{\Omega_{i, j} \uparrow \Omega_{X_{i}}}(\{\overline{\{j\}}) & =m^{\Omega_{i, j}}\left(\left\{n_{i, j}\right\}\right) \\ m^{\Omega_{i, j} \uparrow \Omega_{X_{i}}}\left(\Omega_{X_{i}}\right) & =m^{\Omega_{i, j}}\left(\Omega_{i, j}\right)\end{cases}
$$

In the same manner, mass functions $m^{\Omega_{i, j}}$ can be vacuously extended on $\Omega_{Y_{j}}$ for all $(i, j) \in I \times J$ :

$$
\begin{cases}m^{\Omega_{i, j} \uparrow \Omega_{Y_{j}}}(\{i\}) & =m^{\Omega_{i, j}}\left(\left\{y_{i, j}\right\}\right) \\ m^{\Omega_{i, j} \uparrow \Omega_{Y_{j}}}(\overline{\{i\}}) & =m^{\Omega_{i, j}}\left(\left\{n_{i, j}\right\}\right) \\ m^{\Omega_{i, j} \uparrow \Omega_{Y_{j}}}\left(\Omega_{Y_{j}}\right) & =m^{\Omega_{i, j}}\left(\Omega_{i, j}\right)\end{cases}
$$

In the following, mass functions $m^{\Omega_{i, j} \uparrow \Omega_{X_{i}}}$ (respectively $m^{\Omega_{i, j} \uparrow \Omega_{Y_{j}}}$ ) are denoted $m_{j}^{\Omega_{X_{i}}}$ (respectively $m_{i}^{\Omega_{Y_{j}}}$ ).

\subsection{Combining mass functions}

At this level:

- for each $i \in I=\{1, \ldots, N\}, M$ mass functions $m_{j}^{\Omega_{X_{i}}}$ have been created regarding the association of each object $X_{i}$ toward the $Y_{j}$, the focal elements of each one being $\{j\}, \overline{\{j\}}$, and $\Omega_{X_{i}}$.

- for each $j \in J=\{1, \ldots, M\}, N$ mass functions $m_{i}^{\Omega_{Y_{j}}}$ have been created regarding the association of each object $Y_{j}$ toward the $X_{i}$, the focal elements of each one being $\{i\}, \overline{\{i\}}$, et $\Omega_{Y_{j}}$.

The $M$ mass functions $m_{j}^{\Omega_{X_{i}}}$, considered as distinct and reliable, are combined using the conjunctive rule of combination (6). 
Let us denote $m^{\Omega_{X_{i}}}$ the resulting mass function:

$$
m^{\Omega_{X_{i}}}=\bigcirc_{j \in J} m_{j}^{\Omega_{X_{i}}} .
$$

For all $k \in J$ :

$$
m^{\Omega_{X_{i}}}(\{k\})=\sum_{\cap A_{j}=\{k\}} \prod_{j \in J} m_{j}^{\Omega_{X_{i}}}\left(A_{j}\right),
$$

where, for all $j \in J, A_{j}=\{j\}, \overline{\{j\}}$, or $\Omega_{X_{i}}$.

But:

$$
\begin{aligned}
\cap_{j \in J} A_{j}=\{k\} \Leftrightarrow & A_{k}=\{k\} \text { and }\left(A_{j}=\overline{\{j\}}\right. \text { or } \\
& \left.A_{j}=\Omega_{X_{i}}, \forall j \in J \backslash\{k\}\right), \\
\Leftrightarrow & A_{k}=\{k\} \text { and } \\
& A_{j} \neq\{j\}, \forall j \in J \backslash\{k\} .
\end{aligned}
$$

Thus, for all $k \in J$ :

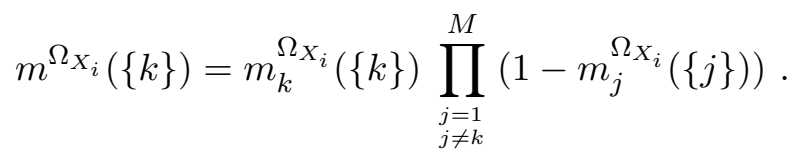

Similarly, for all $K \subseteq J$ :

$$
\begin{aligned}
m^{\Omega_{X_{i}}(\bar{K})} & =\sum_{\cap A_{j}=\bar{K}} \prod_{j \in J} m_{j}^{\Omega_{X_{i}}}\left(A_{j}\right), \\
& =\prod_{j \in K} m_{j}^{\Omega_{X_{i}}}(\overline{\{j\}}) \prod_{j \in \bar{K}} m_{j}^{\Omega_{X_{i}}}\left(\Omega_{X_{i}}\right) .
\end{aligned}
$$

In particular:

$$
\begin{aligned}
& m^{\Omega_{X_{i}}(\{\star\})}=m^{\Omega_{X_{i}}}(\bar{J})=\prod_{j \in J} m_{j}^{\Omega_{X_{i}}}(\overline{\{j\}}), \\
& m^{\Omega_{X_{i}}}\left(\Omega_{X_{i}}\right)=m^{\Omega_{X_{i}}(\bar{\emptyset})}=\prod_{j \in J} m_{j}^{\Omega_{X_{i}}}\left(\Omega_{X_{i}}\right) .
\end{aligned}
$$

At last:

$$
\begin{aligned}
m^{\Omega_{X_{i}}(\emptyset)} & =\sum_{\cap A_{j}=\emptyset} \prod_{j \in J} m_{j}^{\Omega_{X_{i}}}\left(A_{j}\right) \\
& =\sum_{\substack{j, k \in J \\
j \neq k}} m_{j}^{\Omega_{X_{i}}}(\{j\}) m_{k}^{\Omega_{X_{i}}}(\{k\}) .
\end{aligned}
$$


In the same manner, the $N$ mass functions $m_{i}^{\Omega_{Y_{j}}}$ can also be conjunctively combined to result in a mass function $m^{\Omega_{Y_{j}}}$.

Example 1. Let us consider one perceived object $X_{1}$ and two known objects $Y_{1}$ and $Y_{2}$ s.t.:

$$
\left\{\begin{array} { l } 
{ m ^ { \Omega _ { 1 , 1 } } ( \{ y _ { 1 , 1 } \} ) = . 2 } \\
{ m ^ { \Omega _ { 1 , 1 } } ( \{ n _ { 1 , 1 } \} ) = . 4 5 } \\
{ m ^ { \Omega _ { 1 , 1 } ( \Omega _ { 1 , 1 } ) } = . 3 5 }
\end{array} \quad \left\{\begin{array}{l}
m^{\Omega_{1,2}}\left(\left\{y_{1,2}\right\}\right)=.45 \\
m^{\Omega_{1,2}}\left(\left\{n_{1,2}\right\}\right)=.15 \\
m^{\Omega_{1,2}\left(\Omega_{1,2}\right)}=.4
\end{array}\right.\right.
$$

By expressing this information on $\Omega_{X_{1}}$ ( $X_{1}$ 's point of view: with which known object, the perceived object $X_{1}$ is associated? In other words: Where does $X_{1}$ come from?), it is obtained:

$$
\left\{\begin{array} { l } 
{ m _ { 1 } ^ { \Omega _ { X _ { 1 } } } ( \{ 1 \} ) = . 2 } \\
{ m _ { 1 } ^ { \Omega _ { X _ { 1 } } } ( \overline { \{ 1 \} } ) = . 4 5 } \\
{ m _ { 1 } ^ { \Omega _ { X _ { 1 } } } ( \Omega _ { X _ { 1 } } ) = . 3 5 }
\end{array} \quad \left\{\begin{array}{c}
m_{2}^{\Omega_{X_{1}}}(\{2\})=.45 \\
m_{2}^{\Omega_{X_{1}}}(\overline{\{2\}})=.15 \\
m_{2}^{\Omega_{X_{1}}}\left(\Omega_{X_{1}}\right)=.4
\end{array}\right.\right.
$$

The conjunctive combination of $m_{1}^{\Omega_{X_{1}}}$ and $m_{2}^{\Omega_{X_{1}}}$ provides the following result:

$$
\begin{aligned}
& m^{\Omega_{X_{1}}(\{1\})=.2 \times(1-.45)}=.2 \times .55=.11 \\
& m^{\Omega_{X_{1}}(\{2\})}=.45 \times(1-.2)=.45 \times .8=.36 \\
& m^{\Omega_{X_{1}}(\overline{\{1\}})}=m^{\Omega_{X_{1}}(\{2, \star\})}=.45 \times .4=.18 \\
& m^{\Omega_{X_{1}}(\overline{\{2\}})}=m^{\Omega_{X_{1}}(\{1, \star\})}=.15 \times .35=.05 \\
& m^{\Omega_{X_{1}}}(\overline{\{1,2\}})=m^{\Omega_{X_{1}}(\{\star\})}=.45 \times .15=.07 \\
& m^{\Omega_{X_{1}}}\left(\Omega_{X_{1}}\right)=m^{\Omega_{X_{1}}}(\{1,2, \star\})=.35 \times .4=.14
\end{aligned}
$$

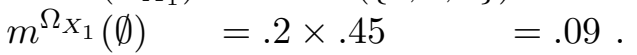

\subsection{Decision-making}

The pignistic probability $\operatorname{Bet} P^{\Omega_{X_{i}}}$ (9) computed from $m^{\Omega_{X_{i}}}$ is defined for all $\omega \in \Omega_{X_{i}}$ by:

$$
\operatorname{BetP}^{\Omega_{X_{i}}(\{\omega\})=} \sum_{\left\{A \subseteq \Omega_{X_{i}}, \omega \in A\right\}} \frac{m^{\Omega_{X_{i}}(A)}}{|A|\left(1-m^{\left.\Omega_{X_{i}}(\emptyset)\right)}\right.} .
$$


Then, for all $k \in J$ :

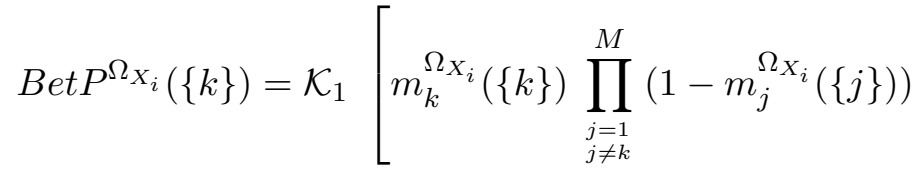

$$
\begin{aligned}
& \left.+\sum_{\substack{k \in \bar{K} \\
K \subseteq J}} \frac{1}{|\bar{K}|} \prod_{j \in K} m_{j}^{\Omega_{X_{i}}}(\overline{\{j\}}) \prod_{j \in \bar{K}} m_{j}^{\Omega_{X_{i}}}\left(\Omega_{X_{i}}\right)\right],
\end{aligned}
$$

where

$$
\mathcal{K}_{1}=\frac{1}{1-m^{\Omega_{X_{i}}(\emptyset)}}=\frac{1}{1-\sum_{\substack{j, k \in J \\ j \neq k}} m_{j}^{\Omega_{X_{i}}}(\{j\}) m_{k}^{\Omega_{X_{i}}}(\{k\})} .
$$

And:

$$
\operatorname{BetP}^{\Omega_{X_{i}}(\{\star\})=} \quad \mathcal{K}_{1} \sum_{K \subseteq J} \frac{1}{|\bar{K}|} \prod_{j \in K} m_{j}^{\Omega_{X_{i}}}(\overline{\{j\}}) \prod_{j \in \bar{K}} m_{j}^{\Omega_{X_{i}}}\left(\Omega_{X_{i}}\right)
$$

Once the pignistic probabilities $B e t P^{\Omega_{X_{i}}}$ computed for each $i \in I$, the chosen decision is the one that maximizes the pignistic probability associated to the joint law $\operatorname{Bet} P^{\Omega_{X_{1}} \times \ldots \times \Omega_{X_{N}}}$ which verifies the constraints expressed in Section 3.1.

This decision-making process is summed up by the following algorithm 1 .

Similarly, an equivalently justified solution consists in computing the decision from the $Y_{j}$ points of view, by maximizing the pignistic probability $\operatorname{Bet}^{\Omega_{Y_{1}} \times \ldots \times \Omega_{Y_{M}}}$.

Example 2 (Example 1 continued). Let us consider again one perceived object $X_{1}$ and two known objects $Y_{1}$ and $Y_{2}$ with the information represented by the BBAs $m^{\Omega_{1,1}}$ and $m^{\Omega_{1,2}}$ defined by Equation 19 .

From $X_{1}$ 's point of view, the conjunctive combination of $m_{1}^{\Omega_{X_{1}}}$ and $m_{2}^{\Omega_{X_{1}}}$ has been detailed in Example 1. The pignistic probability Bet $P^{\Omega_{X_{1}}}$ regarding the association of $X_{1}$ is then given by:

\begin{tabular}{c|ccccccc}
\hline \hline$A$ & $\emptyset$ & $\{1\}$ & $\{2\}$ & $\{\star\}$ & $\{1, \star\}$ & $\{2, \star\}$ & $\{1,2, \star\}$ \\
\hline$m^{\Omega_{X_{1}}(A)}$ & .09 & .11 & .36 & .07 & .05 & .18 & .14 \\
\hline $\operatorname{BetP}^{\Omega_{X_{1}}(A)}$ & & .20 & .55 & .25 & .45 & .80 & 1
\end{tabular}

Conclusion from $X_{1}$ 's point of view: 


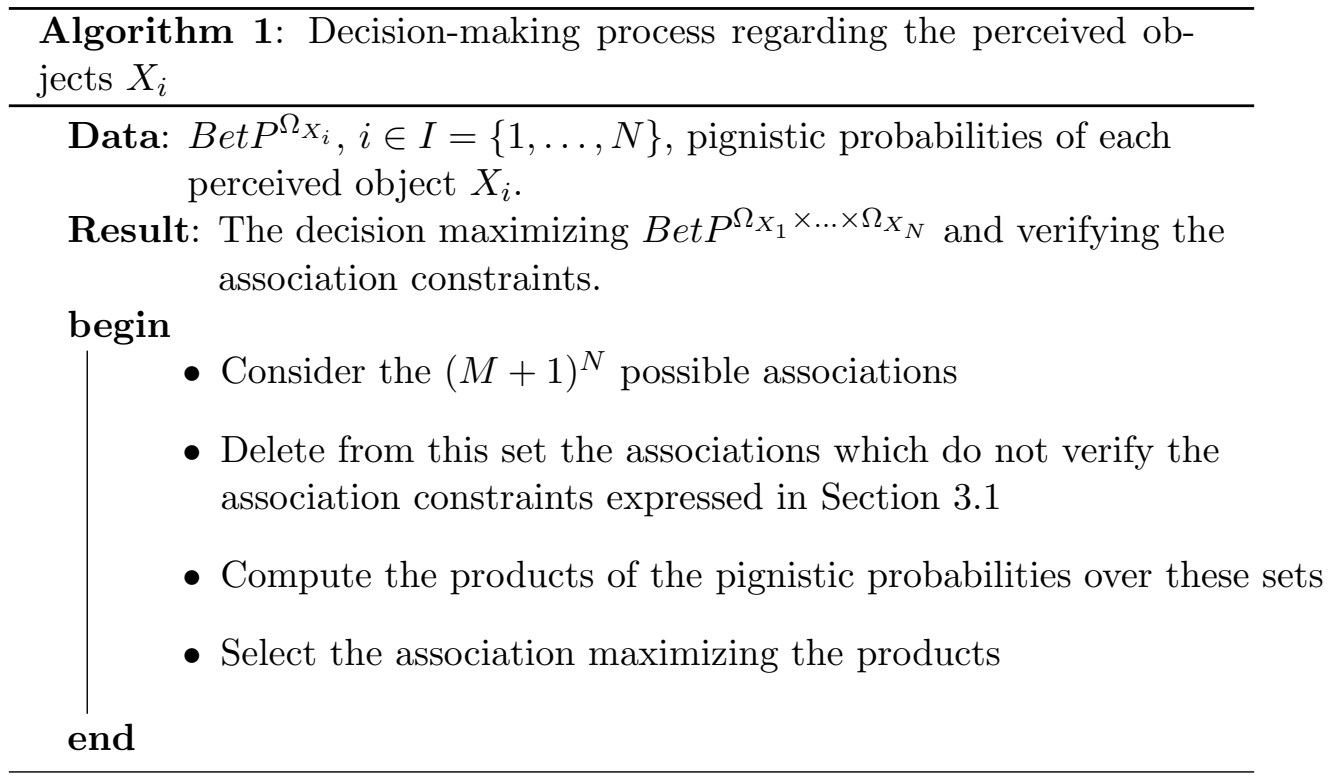

1. The singleton maximizing $\operatorname{Bet}^{\Omega_{X_{1}}}$ is $\{2\}$, so $X_{1}$ is associated with $Y_{2}$;

2. knowing that $Y_{1}$ is not associated, $Y_{1}$ has disappeared (or is hidden).

On the other hand, it is also possible to express the available information on $\Omega_{Y_{1}}$ and $\Omega_{Y_{2}}$ :

$$
\left\{\begin{array} { l l } 
{ m _ { 1 } ^ { \Omega _ { Y _ { 1 } } } ( \{ 1 \} ) } & { = . 2 } \\
{ m _ { 1 } ^ { \Omega _ { Y _ { 1 } } } ( \overline { \{ 1 \} } ) } & { = . 4 5 } \\
{ m _ { 1 } ^ { \Omega _ { Y _ { 1 } } } ( \Omega _ { Y _ { 1 } } ) } & { = . 3 5 }
\end{array} \quad \left\{\begin{array}{c}
m_{1}^{\Omega_{Y_{2}}}(\{1\})=.45 \\
m_{1}^{\Omega_{Y_{2}}}(\overline{\{1\}})=.15 \\
m_{1}^{\Omega_{Y_{2}}}\left(\Omega_{Y_{2}}\right)=.4
\end{array}\right.\right.
$$

As there is only one perceived object $X_{1}$, no combination is necessary:

\begin{tabular}{c|cccc}
\hline \hline$A$ & $\emptyset$ & $\{1\}$ & $\{\star\}$ & $\{1, \star\}$ \\
\hline$m^{\Omega_{Y_{1}}}(A)$ & .2 & .45 & .35 \\
\hline $\operatorname{Bet}^{\Omega_{Y_{1}}}(A)$ & .375 & .625 & 1 \\
\hline$m^{\Omega_{Y_{2}}}(A)$ & .45 & .15 & .4 \\
\hline $\operatorname{BetP}^{\Omega_{Y_{2}}(A)}$ & .65 & .35 & 1 \\
\hline
\end{tabular}

From the association constraints (Section 3.1), the known objects $\left(Y_{1}, Y_{2}\right)$ can be associated to $(1, \star),(\star, 1)$, or $(\star, \star)$.

And, as: 
- $\operatorname{Bet} P^{\Omega_{Y_{1}} \times \Omega_{Y_{2}}}(\{1, \star\})=.375 \times .35=.131 ;$

- $\operatorname{Bet} P^{\Omega_{Y_{1}} \times \Omega_{Y_{2}}}(\{\star, 1\})=.625 \times .65=.406 ;$

- $\operatorname{Bet} P^{\Omega_{Y_{1}} \times \Omega_{Y_{2}}}(\{\star, \star\})=.625 \times .35=.219$,

then Bet $P^{\Omega_{Y_{1}} \times \Omega_{Y_{2}}}$ reaches its valid maximum at $\{\star, 1\}$, so $\left(Y_{1}, Y_{2}\right)$ is associated with $(\star, 1)$; in other words, $Y_{1}$ has disappeared and $Y_{2}$ is associated with $X_{1}$.

In the previous example, the decision coming from $X_{1}$ and the decision coming from the $Y_{j}$ are the same.

Unfortunately, as illustrated by the following Example 3, the decision providing by the criteria of maximizing the joint pignistic probability can be different depending on which point of view (perceived objects $X_{i}$ or known objects $Y_{j}$ ) it is computed.

Example 3. Let us considered one perceived object $X_{1}$, and two known objects $Y_{1}$ and $Y_{2}$, s.t.:

$$
\left\{\begin{array} { l } 
{ m ^ { \Omega _ { 1 , 1 } } ( \{ y _ { 1 , 1 } \} ) = . 5 } \\
{ m ^ { \Omega _ { 1 , 1 } } ( \{ n _ { 1 , 1 } \} ) = 0 } \\
{ m ^ { \Omega _ { 1 , 1 } } ( \Omega _ { 1 , 1 } ) = . 5 }
\end{array} \quad \left\{\begin{array}{l}
m^{\Omega_{1,2}}\left(\left\{y_{1,2}\right\}\right)=.7 \\
m^{\Omega_{1,2}}\left(\left\{n_{1,2}\right\}\right)=.3 \\
m^{\Omega_{1,2}}\left(\Omega_{1,2}\right)=0
\end{array}\right.\right.
$$

By expressing the beliefs on the frames $\Omega_{X_{i}}$ :

$$
\left\{\begin{array} { l } 
{ m _ { 1 } ^ { \Omega _ { X _ { 1 } } } ( \{ 1 \} ) = . 5 } \\
{ m _ { 1 } ^ { \Omega _ { X _ { 1 } } } ( \overline { \{ 1 \} } ) = 0 } \\
{ m _ { 1 } ^ { \Omega _ { X _ { 1 } } } ( \Omega _ { X _ { 1 } } ) = . 5 }
\end{array} \quad \left\{\begin{array}{l}
m_{2}^{\Omega_{X_{1}}}(\{2\})=.7 \\
m_{2}^{\Omega_{X_{1}}}(\overline{\{2\}})=.3 \\
m_{2}^{\Omega_{X_{1}}}\left(\Omega_{X_{1}}\right)=0
\end{array}\right.\right.
$$

the following results are obtained:

\begin{tabular}{c|ccccccc}
\hline \hline$A$ & $\emptyset$ & $\{1\}$ & $\{2\}$ & $\{\star\}$ & $\{1, \star\}$ & $\{2, \star\}$ & $\{1,2, \star\}$ \\
\hline$m^{\Omega_{X_{1}}(A)}$ & .35 & .15 & .35 & 0 & .15 & 0 & 0 \\
\hline $\operatorname{Bet}^{\Omega_{X_{1}}(A)}$ & & .35 & .54 & .11 & .46 & .65 & 1 \\
\hline
\end{tabular}

Then, regarding object $X_{1}$ :

- $X_{1}$ is associated with $Y_{2}$,

- $Y_{1}$ has disappeared. 
From $Y_{1}$ 's and $Y_{2}$ 's points of view:

$$
\left\{\begin{array} { l l } 
{ m _ { 1 } ^ { \Omega _ { Y _ { 1 } } } ( \{ 1 \} ) } & { = . 5 } \\
{ m _ { \Omega _ { 1 } } ( \overline { \{ 1 \} } ) } & { = 0 } \\
{ \Omega _ { Y _ { 1 } } ( \Omega _ { Y _ { 1 } } ) } & { = . 5 }
\end{array} \quad \left\{\begin{array}{c}
m_{1}^{\Omega_{Y_{2}}}(\{1\})=.7 \\
m_{1} \Omega_{Y_{2}}(\overline{\{1\}})=.3 \\
m_{1}^{\Omega_{Y_{2}}}\left(\Omega_{Y_{2}}\right)=0 .
\end{array}\right.\right.
$$

So:

\begin{tabular}{c|cc}
\hline \hline$A$ & $\{1\}$ & $\{\star\}$ \\
\hline $\operatorname{Bet}^{\Omega_{Y_{1}}}$ & .75 & .25 \\
$\operatorname{Bet} P^{\Omega_{Y_{2}}}$ & .70 & .30 \\
\hline
\end{tabular}

As $.75 \times .3>.7 \times .25$, Bet $P^{\Omega_{Y_{1}} \times \Omega_{Y_{2}}}$ reaches its valid maximum at $\{1, \star\}$, which implies that:

- $Y_{1}$ is associated with $X_{1}$,

- $Y_{2}$ has disappeared.

This decision is then different from the previous one.

Let us also remark that the introduction of a rejection decision, as presented in Section 2.3, can also imply a different decision according to the $X_{i}$ or $Y_{j}$ points of view. For instance, by choosing $c_{0}$ equal to 0.5 in the Example 2, from $X_{1}$ the same decision is made as $\operatorname{Bet}^{\Omega_{X_{1}}}(\{2\}) \geq 1-c_{0}$, however as $\operatorname{Bet} P^{\Omega_{Y_{1}} \times \Omega_{Y_{2}}}(\{\star, 1\})<1-c_{0}$, the decision made according to the $Y_{j}$ is $d_{0}$ (a rejection).

A practical solution consists in choosing a decision by favouring either the perceived objects or the known objects.

However, to relativize this problem, conflicting decisions happen in very few cases in the particular application described in Section 5, less than 1\% of the cases in this example.

\section{Discussion}

\subsection{What's new in comparison to Rombaut and Gruyer's approaches?}

The approach presented in this article differs mainly from Rombaut and Gruyer's approaches $[23,16]$ by regarding two points:

1. the combination of BBAs $m_{j}^{\Omega_{X_{i}}}=m^{\Omega_{i, j} \uparrow \Omega_{X_{i}}}$ and $m_{i}^{\Omega_{Y_{j}}}=m^{\Omega_{i, j} \uparrow \Omega_{Y_{j}}}$;

2. the decision-making process. 
In both Rombaut's approach [23] and Gruyer's approach [16], BBAs $m_{j}^{\Omega_{X_{i}}}$ and $m_{i}^{\Omega_{Y_{j}}}$ are not classically conjunctively combined with (14). To simplify the combination and to make it computationally efficient, it is proposed to allocate masses only on singletons and the universe. Thus the following mergers are proposed, $\forall i \in I$ :

$$
\begin{aligned}
& m_{\text {Rombaut }}^{\Omega_{X_{i}}}(\{\emptyset\})=m^{\Omega_{X_{i}}(\{\emptyset\})} \\
& m_{\text {Rombaut }}^{\Omega_{X_{i}}}(\{k\})=m^{\Omega_{X_{i}}}(\{k\}), \quad \forall k \in J, \\
& m_{\substack{\text { Rombaut }_{X_{i}} \\
\Omega_{X_{X_{i}}}}}(\{\star\})=m^{\Omega_{X_{i}}(\{\star\})}
\end{aligned}
$$

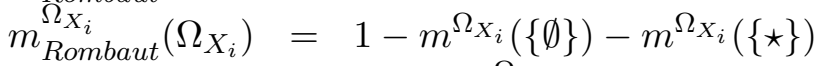

$$
\begin{aligned}
& -\sum_{k \in J} m^{\Omega_{X_{i}}}(\{k\}) \text {. }
\end{aligned}
$$

In [16], the authors suggest a decision-making system based on BBAs $m^{\Omega_{X_{i}}}$ and $m^{\Omega_{Y_{j}}}$ whose focal elements, thanks to Rombaut's combination, are either a singleton or the universe. In outline:

- An association matrix $N \times M$ is built such that each of its elements $(i, j)$ is equal to the product $m^{\Omega_{X_{i}}}(\{j\}) \times m^{\Omega_{Y_{j}}}(\{i\})$. Each row $i$ is then associated with a perceived object $X_{i}$, and each column $j$ is associated with a known object $Y_{j}$.

- If necessary, fictive objects are added to make the latter matrix squared.

- A coupling algorithm, the Hungarian algorithm, is then applied to this matrix, this latter algorithm providing an optimal decision regarding the sum of the beliefs.

- A final treatment deals with the objects appearance.

Applied to the examples presented in [23] and [16], the model presented in this article and Gruyer's approach lead to the same results.

Example 4 (Rombaut [23]). Let us consider two perceived objects $X_{i}$ and two known objects $Y_{j}$, s.t.:

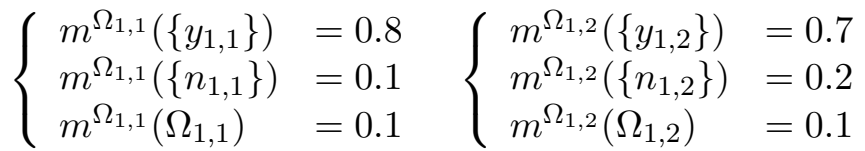

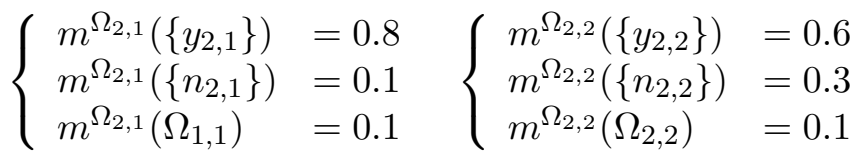


By expressing these beliefs over $\Omega_{X_{1}}$ and $\Omega_{X_{2}}$, it is obtained:

$$
\left\{\begin{array} { l } 
{ m _ { 1 } ^ { \Omega _ { X _ { 1 } } } ( \{ 1 \} ) = 0 . 8 } \\
{ m _ { 1 } \Omega _ { X _ { 1 } } ( \overline { \{ 1 \} } ) = 0 . 1 } \\
{ m _ { 1 } ^ { \Omega _ { X _ { 1 } } } ( \Omega _ { X _ { 1 } } ) = 0 . 1 }
\end{array} \left\{\begin{array} { c } 
{ m _ { 2 } ^ { \Omega _ { X _ { 1 } } } ( \{ 2 \} ) = 0 . 7 } \\
{ m _ { 2 } ^ { \Omega _ { X _ { 1 } } } ( \overline { \{ 2 \} } ) = 0 . 2 } \\
{ m _ { 2 } ^ { \Omega _ { X _ { 1 } } } ( \Omega _ { X _ { 1 } } ) = 0 . 1 }
\end{array} \left\{\begin{array} { c } 
{ m _ { 1 } ^ { \Omega _ { X _ { 2 } } } ( \{ 1 \} ) = 0 . 8 } \\
{ m _ { 1 } ( \Omega _ { X _ { 2 } } ( \overline { \{ 1 \} } ) = 0 . 1 } \\
{ m _ { 1 } ^ { \Omega _ { X _ { 2 } } } ( \Omega _ { X _ { 2 } } ) = 0 . 1 }
\end{array} \left\{\begin{array}{c}
m_{2}^{\Omega_{X_{2}}}(\{2\})=0.6 \\
m_{2} \Omega_{X_{2}}(\overline{\{2\}})=0.3 \\
m_{2}^{\Omega_{X_{2}}}\left(\Omega_{X_{2}}\right)=0.1
\end{array}\right.\right.\right.\right.
$$

Pignistic probabilities are then defined by:

\begin{tabular}{c|ccc}
\hline \hline$A$ & $\{1\}$ & $\{2\}$ & $\{\star\}$ \\
\hline $\operatorname{Bet} P^{\Omega_{X_{1}}}$ & 0.57 & 0.34 & 0.09 \\
$\operatorname{Bet} P^{\Omega_{X_{2}}}$ & 0.65 & 0.25 & 0.10 \\
\hline
\end{tabular}

Thus Bet $P^{\Omega_{X_{1}} \times \Omega_{X_{2}}}$ reaches its valid maximum at $\{2,1\}$, and regarding the $X_{i}$ :

1. $X_{1}$ is associated with $Y_{2}$;

2. $X_{2}$ is associated with $Y_{1}$;

By expressing the beliefs regarding objects $Y_{j}$, we have:

$$
\left\{\begin{array} { l } 
{ m _ { 1 } ^ { \Omega _ { Y _ { 1 } } } ( \{ 1 \} ) = 0 . 8 } \\
{ m _ { 1 } ^ { \Omega _ { Y _ { 1 } } } ( \overline { \{ 1 \} } ) = 0 . 1 } \\
{ m _ { 1 } ^ { \Omega _ { Y _ { 1 } } } ( \Omega _ { Y _ { 1 } } ) = 0 . 1 }
\end{array} \left\{\begin{array} { c } 
{ m _ { 2 } ^ { \Omega _ { Y _ { 1 } } } ( \{ 2 \} ) = 0 . 8 } \\
{ m _ { 2 } ^ { \Omega _ { Y _ { 1 } } } ( \overline { \{ 2 \} } ) = 0 . 1 } \\
{ m _ { 2 } ^ { \Omega _ { Y _ { 1 } } } ( \Omega _ { Y _ { 1 } } ) = 0 . 1 }
\end{array} \left\{\begin{array} { c } 
{ m _ { 1 } ^ { \Omega _ { Y _ { 2 } } } ( \{ 1 \} ) = 0 . 7 } \\
{ m _ { 1 } \Omega _ { Y _ { 2 } } ( \overline { \{ 1 \} } ) = 0 . 2 } \\
{ m _ { 1 } ^ { \Omega _ { Y _ { 2 } } } ( \Omega _ { Y _ { 2 } } ) = 0 . 1 }
\end{array} \left\{\begin{array}{c}
m_{2}^{\Omega_{Y_{2}}}(\{2\})=0.6 \\
m_{2} \Omega_{Y_{2}}(\overline{\{2\}})=0.3 \\
m_{2}^{\Omega_{Y_{2}}}\left(\Omega_{Y_{2}}\right)=0.1
\end{array}\right.\right.\right.\right.
$$

Then:

\begin{tabular}{c|ccc}
\hline \hline$A$ & $\{1\}$ & $\{2\}$ & $\{\star\}$ \\
\hline $\operatorname{Bet}^{\Omega_{Y_{1}}}$ & 0.47 & 0.47 & 0.06 \\
$\operatorname{Bet} P^{\Omega_{Y_{2}}}$ & 0.52 & 0.33 & 0.15 \\
\hline
\end{tabular}

Conclusion from $Y_{1}$ 's and $Y_{2}$ 's point of view:

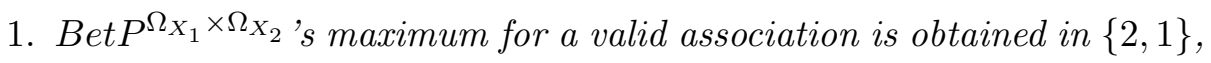
then $Y_{1}$ is associated with $X_{2}$, and $Y_{2}$ is associated with $X_{1}$.

Example 5 (Gruyer [16]). In this example, three perceived objects $\left(X_{i}\right)$ 
and four objects $\left(Y_{j}\right)$ are considered such that:

$$
\begin{aligned}
& \left\{\begin{array} { l } 
{ m ^ { \Omega _ { 1 , 1 } } ( \{ y _ { 1 , 1 } \} ) = 0 . 8 0 } \\
{ m ^ { \Omega _ { 1 , 1 } } ( \{ n _ { 1 , 1 } \} ) = 0 } \\
{ m ^ { \Omega _ { 1 , 1 } } ( \Omega _ { 1 , 1 } ) = 0 . 2 0 }
\end{array} \left\{\begin{array} { l } 
{ m ^ { \Omega _ { 1 , 2 } } ( \{ y _ { 1 , 2 } \} ) = 0 } \\
{ m ^ { \Omega _ { 1 , 2 } } ( \{ n _ { 1 , 2 } \} ) = 0 . 9 9 } \\
{ m ^ { \Omega _ { 1 , 2 } } ( \Omega _ { 1 , 2 } ) = 0 . 0 1 }
\end{array} \left\{\begin{array} { l } 
{ m ^ { \Omega _ { 1 , 3 } } ( \{ y _ { 1 , 3 } \} ) = 0 } \\
{ m ^ { \Omega _ { 1 , 3 } } ( \{ n _ { 1 , 3 } \} ) = 0 . 9 7 } \\
{ m ^ { \Omega _ { 1 , 3 } } ( \Omega _ { 1 , 3 } ) = 0 . 0 3 }
\end{array} \left\{\begin{array}{l}
m^{\Omega_{1,4}}\left(\left\{y_{1,4}\right\}\right)=0 \\
m^{\Omega_{1,4}}\left(\left\{n_{1,4}\right\}\right)=0.99 \\
m^{\Omega_{1,4}}\left(\Omega_{1,4}\right)=0.01
\end{array}\right.\right.\right.\right. \\
& \left\{\begin{array} { l } 
{ m ^ { \Omega _ { 2 , 1 } } ( \{ y _ { 2 , 1 } \} ) = 0 . 5 7 } \\
{ m ^ { \Omega _ { 2 , 1 } } ( \{ n _ { 2 , 1 } \} ) = 0 } \\
{ m ^ { \Omega _ { 2 , 1 } } ( \Omega _ { 2 , 1 } ) = 0 . 4 3 }
\end{array} \left\{\begin{array} { l } 
{ m ^ { \Omega _ { 2 , 2 } } ( \{ y _ { 2 , 2 } \} ) = 0 . 5 7 } \\
{ m ^ { \Omega _ { 2 , 2 } } ( \{ n _ { 2 , 2 } \} ) = 0 } \\
{ m ^ { \Omega _ { 2 , 2 } } ( \Omega _ { 2 , 2 } ) = 0 . 4 3 }
\end{array} \quad \left\{\begin{array} { l } 
{ m ^ { \Omega _ { 2 , 3 } } ( \{ y _ { 2 , 3 } \} ) = 0 } \\
{ m ^ { \Omega _ { 2 , 3 } } ( \{ n _ { 2 , 3 } \} ) = 0 . 5 2 } \\
{ m ^ { \Omega _ { 2 , 3 } } ( \Omega _ { 2 , 3 } ) = 0 . 4 8 }
\end{array} \left\{\begin{array}{l}
m^{\Omega_{2,4}}\left(\left\{y_{2,4}\right\}\right)=0 \\
m^{\Omega_{2,4}}\left(\left\{n_{2,4}\right\}\right)=0.99 \\
m^{\Omega_{2,4}}\left(\Omega_{2,4}\right)=0.01
\end{array}\right.\right.\right.\right. \\
& \left\{\begin{array} { l } 
{ m ^ { \Omega _ { 3 , 1 } } ( \{ y _ { 3 , 1 } \} ) = 0 } \\
{ m ^ { \Omega _ { 3 , 1 } } ( \{ n _ { 3 , 1 } \} ) = 0 . 9 9 } \\
{ m ^ { \Omega _ { 3 , 1 } } ( \Omega _ { 3 , 1 } ) = 0 . 0 1 }
\end{array} \left\{\begin{array} { l } 
{ m ^ { \Omega _ { 3 , 2 } } ( \{ y _ { 3 , 2 } \} ) = 0 . 6 1 } \\
{ m ^ { \Omega _ { 3 , 2 } } ( \{ n _ { 3 , 2 } \} ) = 0 } \\
{ m ^ { \Omega _ { 3 , 2 } } ( \Omega _ { 3 , 2 } ) = 0 . 3 9 }
\end{array} \quad \left\{\begin{array} { l } 
{ m ^ { \Omega _ { 3 , 3 } } ( \{ y _ { 3 , 3 } \} ) = 0 } \\
{ m ^ { \Omega _ { 3 , 3 } } ( \{ n _ { 3 , 3 } \} ) = 0 . 5 2 } \\
{ m ^ { \Omega _ { 3 , 3 } } ( \Omega _ { 3 , 3 } ) = 0 . 4 8 }
\end{array} \left\{\begin{array}{l}
m^{\Omega_{3,4}}\left(\left\{y_{3,4}\right\}\right)=0 \\
m^{\Omega_{3,4}}\left(\left\{n_{3,4}\right\}\right)=0.99 \\
m^{\Omega_{3,4}}\left(\Omega_{3,4}\right)=0.01
\end{array}\right.\right.\right.\right.
\end{aligned}
$$

Pignistic probabilities regarding objects $X_{i}$ are given by:

\begin{tabular}{c|ccccc}
\hline \hline$A$ & $\{1\}$ & $\{2\}$ & $\{3\}$ & $\{4\}$ & $\{\star\}$ \\
\hline $\operatorname{BetP}^{\Omega_{X_{1}}}$ & 0.90 & 0.00 & 0.00 & 0.00 & 0.10 \\
$\operatorname{Bet}^{\Omega_{X_{2}}}$ & 0.44 & 0.44 & 0.03 & 0.00 & 0.08 \\
$\operatorname{Bet} P^{\Omega_{X_{3}}}$ & 0.00 & 0.77 & 0.06 & 0.00 & 0.16 \\
\hline
\end{tabular}

The association result can be obtained from Algorithm 1. However, when numerous probabilities are equal to zero, the following Algorithm 2 can be employed.

The use of Algorithm 2 on this example is illustrated in Figure 3.

$\operatorname{Bet} P^{\Omega_{X_{1}} \times \Omega_{X_{2}} \times \Omega_{X_{3}}}$ reaches its maximum $.9 \times .08 \times .77$ at $\{1, \star, 2\}$. Thus:

1. $X_{1}$ is associated with $Y_{1}$;

2. $X_{2}$ has appeared;

3. $X_{3}$ is associated with $Y_{2}$;

4. then $Y_{3}$ and $Y_{4}$ have disappeared.

On the other side, by considering $Y_{j} s^{\prime}$ points of view:

\begin{tabular}{c|cccc}
\hline \hline$A$ & $\{1\}$ & $\{2\}$ & $\{3\}$ & $\{\star\}$ \\
\hline $\operatorname{Bet}^{\Omega_{Y_{1}}}$ & 0.69 & 0.26 & 0.00 & 0.05 \\
$\operatorname{Bet} P^{\Omega_{Y_{2}}}$ & 0.00 & 0.42 & 0.49 & 0.09 \\
$\operatorname{Bet}^{\Omega_{Y_{3}}}$ & 0.01 & 0.20 & 0.20 & 0.59 \\
$\operatorname{Bet} P^{\Omega_{Y_{4}}}$ & 0.05 & 0.05 & 0.05 & 0.985 \\
\hline
\end{tabular}

$\operatorname{Bet}^{\Omega_{Y_{1}} \times \Omega_{Y_{2}} \times \Omega_{Y_{3}} \times \Omega_{Y_{4}}}$ reaches its maximum $.69 \times .49 \times .59 \times .985$ at $\{1,3, \star, \star\}$.

Then: 


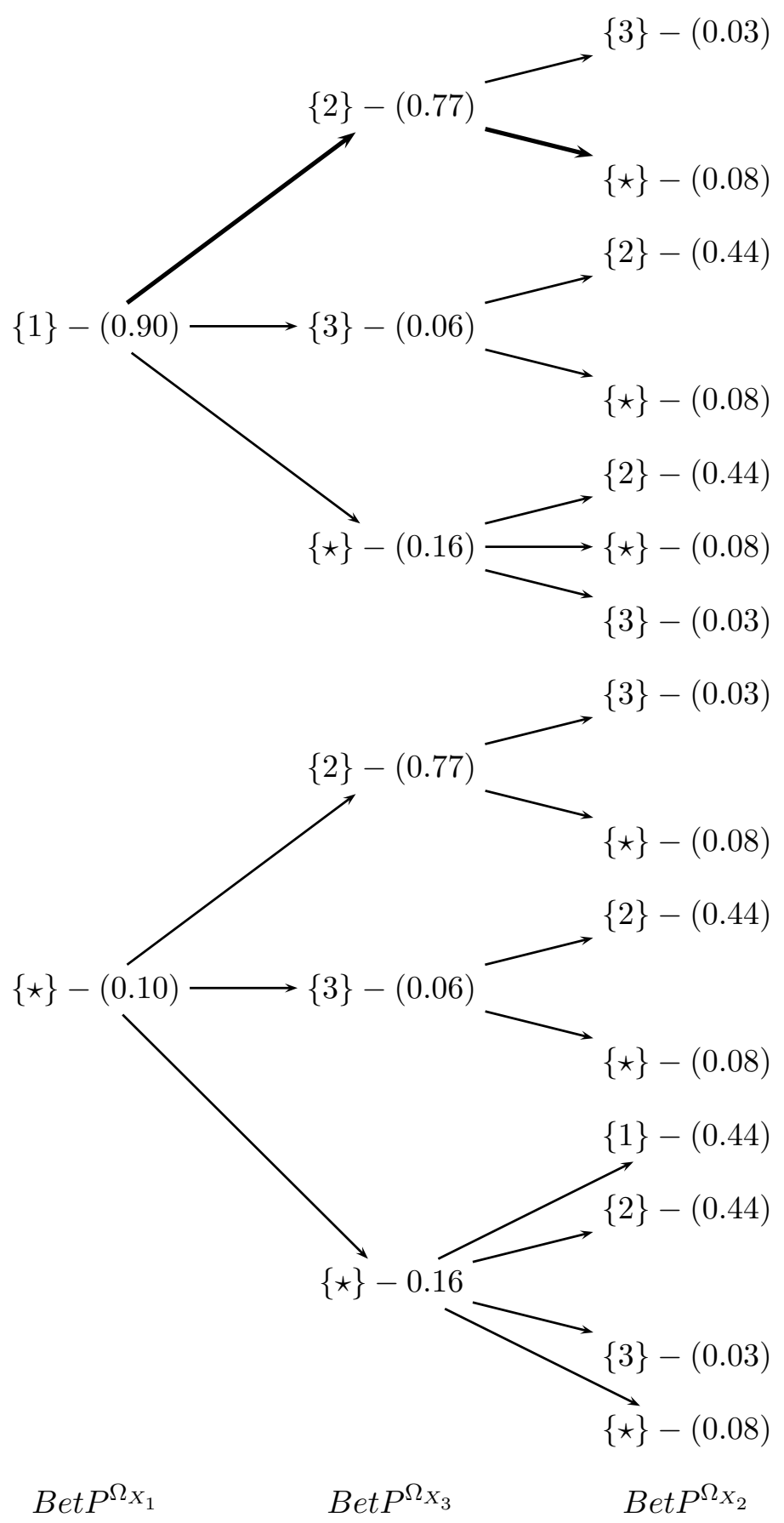

Figure 3: Illustration of the use of Algorithm 2 on Example 5 


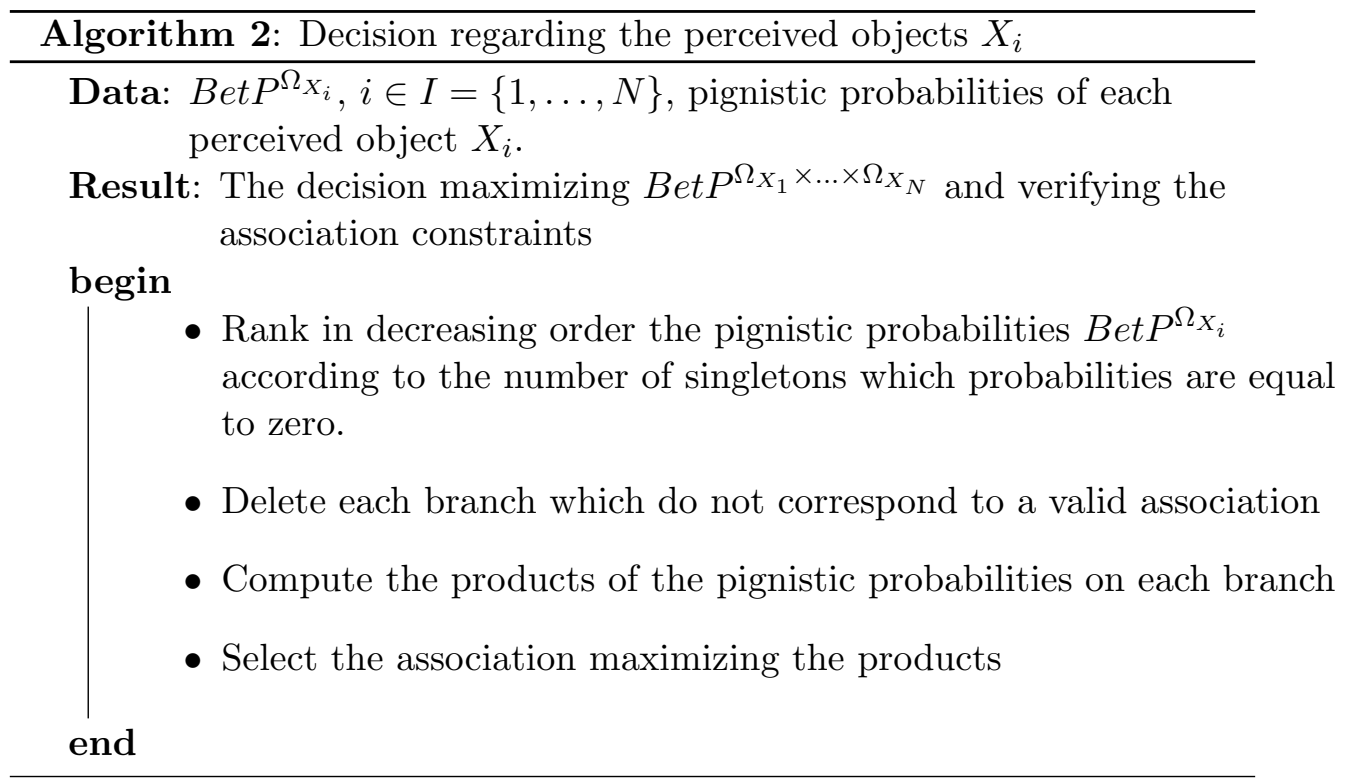

- $Y_{1}$ is associated with $X_{1}$;

- $Y_{2}$ is associated with $X_{3}$;

- $Y_{3}$ has disappeared;

- $Y_{4}$ has disappeared;

- and, $X_{2}$ has appeared.

In this example, the solution proposed by Gruyer in [16] leads to the same conclusion.

\subsection{About Ristic and Smets' approach}

The problem tackled by Ristic and Smets in [22] is somewhat different from the association problem described in this article. Ristic and Smets consider a given volume of interest containing an unknown number of objects. While sensors we consider give information regarding the associations of each object detected at a time step $t$, with previous objects detected at a previous time step $t-1$, Ristic and Smets's sensors provide information regarding the class of each object they have detected in the scene, for instance helicopter, airplane, ... The "association problem" they try to solve consists then in determining the number of objects as well as the class of 
each one. Besides, the appearance and disappearance of objects do not take directly part of their problem. The application of Ristic and Smets' works to our problem is consequently not straightforward.

However, some technical points of this model should be taken into account and investigated.

Following [10], the authors remark that the mass given to the empty set, after conjunctively combining two BBAs expressing themselves on the class of two different objects is equal to the belief that these two objects do not belong to the same class, an idea already present in [1] (multi-sensor fusion for submarine detection) and in [25] (intelligence clustering).

At last, the criteria the authors maximize is based on the plausibility of each possible associations. As justified in [28], the pignistic transformation has been chosen to make the decision in this article. A first investigation in the direction of the plausibility consists in using the plausibility-probability transformation [5].

\section{Results on real data}

In this section, the approach presented in this article (Section 3) is compared to the approach of Rombaut and Gruyer on real data coming from a DV camera placed behind the windshield of a car. This DV camera has a CCD sensor, a $720 \times 576$ pixels resolution, an angle ranging from -0.5 to +0.5 radians (i.e. approximately $\pm 30^{\circ}$ ), and works at 25 images per second $\left(\Delta_{t}=.04 s\right)$, a filmed image example being presented in Figure 4.

The video sequence allowing one to compare the two approaches includes about 3250 images corresponding to a 130-second playing time. Each images contains 1 to 6 objects. The distribution of the number of objects in each image is illustrated in Figure 5. It can be observed that the sequence to analyse is mainly composed of images with 1 object, 2 objects, 3 objects or more.

During the sequence, 75 distinct objects were manually identified as illustrated in Figure 4 (no automatic treatment of the images), the number of associations to realize being equal to 6800 . The ground truth is then 


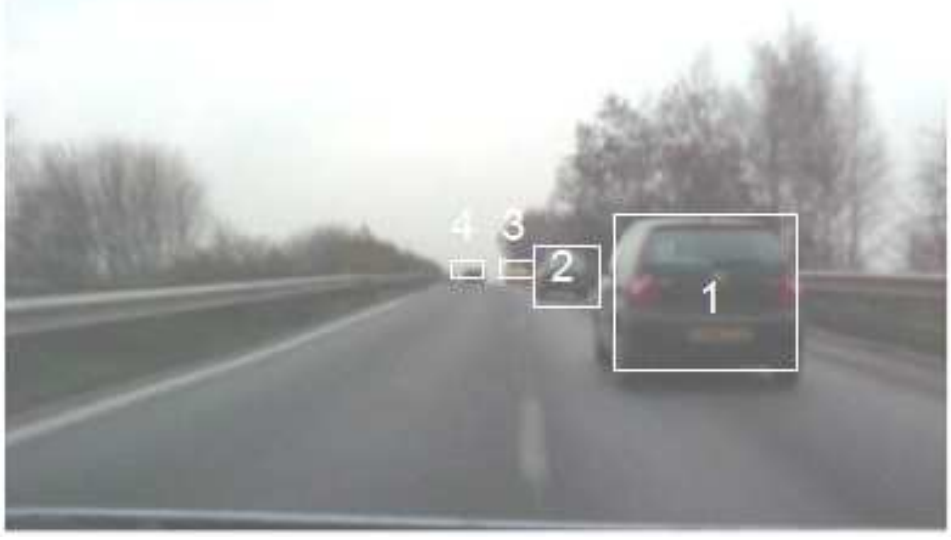

Figure 4: Four vehicles in a selected image in the sequence.

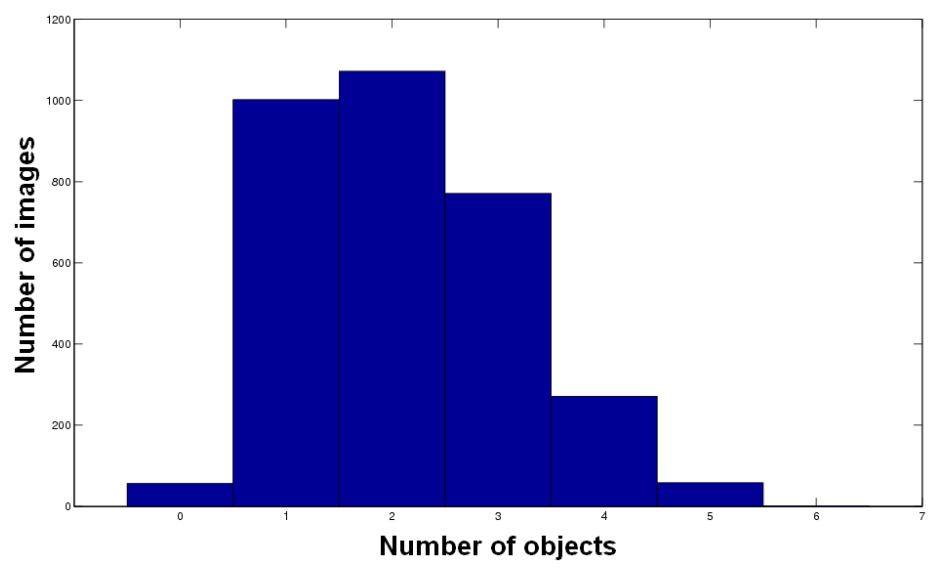

Figure 5: Number of objects in each image during the sequence.

known. It allows us to compute the good recognition rate $(G R R)$ of each approach during this sequence. Formally, this rate is defined as follows:

$$
G R R=\frac{\text { Number of correct associations }}{\text { Number of association to realize }}
$$


In the same manner, the rejection rate $(R R)$ and the error rate $(E R)$ are defined by:

$$
\begin{aligned}
& R R=\frac{\text { Number of rejections }}{\text { Number of association to realize }} \\
& E R=\frac{\text { Number of incorrect associations }}{\text { Number of association to realize }} .
\end{aligned}
$$

The sum of these rates is equal to 1 .

Distance and angle criteria allow the creation of two mass functions $m_{\text {distance }}^{\Omega_{i, j}}$ and $m_{\text {angle }}^{\Omega_{i, j}}$, regarding the association between each perceived object $X_{i}$ and known object $Y_{j}$.

The distance was estimated as a function of the height and the width in pixels of the object observed in the scene thanks to an interpolation method illustrated in Figure 6.

On the other hand, the angle between two objects is computed from the gravity centre of the perceived object in the image (Figure 4).

The measurements provided are very noisy. For instance, there can be a variation of $20 \mathrm{~m}$ for the same object from an image to the next one. Likewise, angle variations can be as high as $100 \%$, from $0.01 r d$ to $0.02 r d$ for two consecutive measurements of the same object.

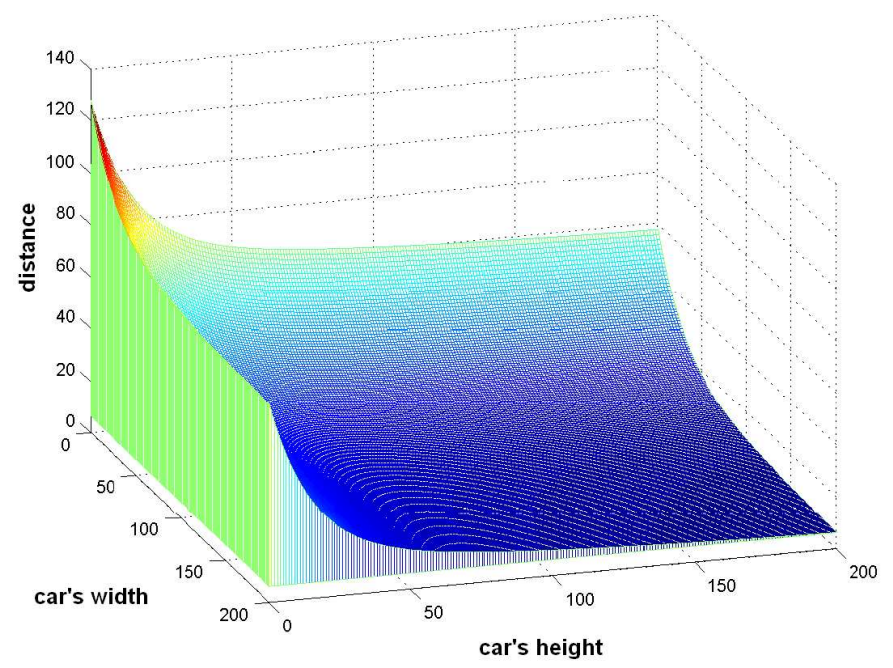

Figure 6: Interpolation function giving the distance in meter depending on the height and the width in pixels of the object in the scene. 
In this application, masses are fixed in the following way:

$$
\begin{cases}m^{\Omega_{i, j}}\left(\left\{y_{i, j}\right\}\right) & =\beta \phi_{i, j}\left(e_{i, j}\right) \\ m^{\Omega_{i, j}}\left(\left\{n_{i, j}\right\}\right) & =\beta\left(1-\phi_{i, j}\left(e_{i, j}\right)\right) \\ m^{\Omega_{i, j}}\left(\Omega_{i, j}\right) & =1-\beta\end{cases}
$$

where:

- $0<\beta<1$ is a constant representing the degree of reliability of the source of information (cf the discounting operation [26, page 252], see $[18,21]$ for other correction mechanisms).

- $\phi_{i, j}($.$) is a monotone decreasing function s.t. \phi_{i}(0)=1$ and $\lim _{e \rightarrow \infty} \phi_{i}(e)=$ 0

- $e_{i, j}$ is the dissimilarity measure between the perceived object $X_{i}$ and the known object $Y_{j}$, which means the difference of distance and the difference of angle in this application.

The function $\phi_{i, j}$ is chosen as follows [8]:

$$
\phi_{i, j}\left(e_{i, j}\right)=\exp ^{\left(-\left(e_{i, j}\right)^{2}\right)} .
$$

Constant $\beta$ being fixed at 0.9 , these two mass functions are combined thanks to the Dempster's rule of combination to obtain a mass function $m^{\Omega_{i, j}}$ :

$$
m^{\Omega_{i, j}}=m_{\text {distance }}^{\Omega_{i, j}} \oplus m_{\text {angle }}^{\Omega_{i, j}} \quad \forall i \in I, \forall j \in J .
$$

The association model presented in Section 3 only need one BBA expressing the information regarding the association between object $X_{i}$ and object $Y_{j}$. In this application, we are lucky enough to have two information sources. Thus these two pieces of information are firstly combined using a well justified rule for the combination of two distinct sources. The choice to combine theses sources at this step, and the choice of the rule have been left for further study.

In Figure 7, the good recognition rate of the two approaches presented in this article obtained in this video sequence is represented as a function of the rejection cost (Section 2.3). It can be observed that as soon as the rejection cost becomes greater than 0 , the good recognition rates obtained with the conjunctive combination are greater than those obtained with Rombaut's combination, which is recalled to be also used in Gruyer's approach. 
Let us note that the decisions have been computed on the basis of the perceived objects. As mentioned in Section 3.4, these decisions are not necessary identical with those computed from the known objects point of view. However, as illustrated in Figure 8, this conflicting decision rate remains very low in this application (from $0 \%$ to less than $1 \%$ depending on the rejection cost). Let us also recall that, as illustrated at the end of Example 2 , the introduction of a rejection cost enhances the appearance of conflicting decisions.

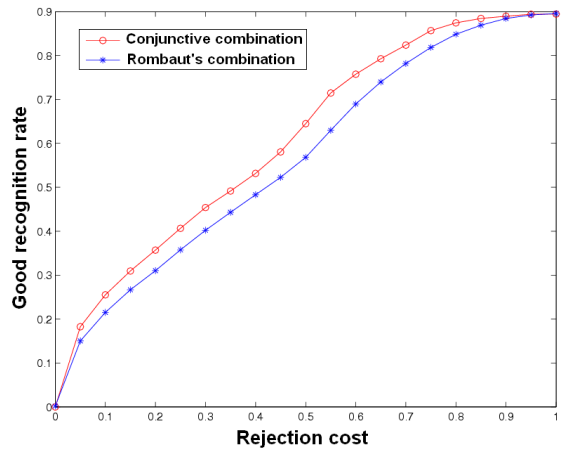

Figure 7: Good recognition rate in function of the rejection cost.

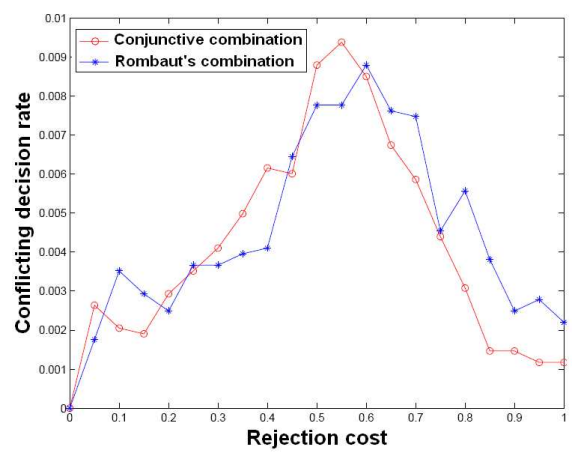

Figure 8: Conflicting decision rate in function of the rejection cost.

\section{Conclusion and prospects}

In this article, a modelling of the association problem in the belief function framework has been presented. It has been shown in particular, how tools from the theory of belief functions such as the vacuous extension, the 
conjunctive combination rule and the pignistic transformation can be applied. Validated on real data, this approach can perform better good recognition rates than Rombaut's initial approach as soon as a rejection cost is introduced.

Concerning the prospects, even if it concerns a reduce number of cases, the resolution of the possible conflicting decisions between perceived and known objects have to be deeper investigated, even if it seems an integral part of this problem.

The decomposition of the BBAs [11] expressing the beliefs regarding the associations between known objects and perceived objects could also be studied in order to use a more adapted rule.

Subsequently, this approach should be enhanced by introducing information coming from the tracking of vehicles at time steps preceding the current analysis.

\section{Acknowledgements}

These works have been financed by the French region Nord-Pas de Calais under the project CISIT (Campus International pour la Sécurité et l'Intermodalité des Transports).

\section{References}

[1] A. Ayoun and Ph. Smets. Data association in multi-target detection using the transferable belief model. International Journal of Intelligent Systems, 16(10):1167-1182, 2001.

[2] Y. Bar-Shalom. Multitarget/Multisensor Tracking: Applications and Advances vol III, Artech House, 2000.

[3] S. Blackman and R. Popoli. Design and Analysis of Modern Tracking Systems, Artech House, 1999.

[4] I. Bloch. Fusion of Information under Imprecision and Uncertainty, Numerical Methods, and Image Information Fusion. Multisensor Data Fusion, A.K. Hyder et al (Eds.), pp. 267-294, 2002.

[5] B.R. Cobb, P.P. Shenoy. On the plausibility transformation method for translating belief function models to probability models. International Journal of Approximate Reasoning, 41(3):314-330, 2006. 
[6] M.H. DeGroot. Optimal Statistical Decisions. McGraw-Hill, New York, 1970.

[7] A. Dempster. A generalization of Bayesian inference. Journal of Royal Statistical Society, B 30:205-247, 1968.

[8] T. Denœux. A k-nearest neighbour classification rule based on Dempster-Shafer theory. IEEE Transactions on Systems, Man and Cybernetics, 25(5):804-813, 1995.

[9] T. Denœux. Analysis of evidence-theoretic decision rules for pattern classification. Pattern Recognition, 30(7):1095-1107, 1997.

[10] T. Denoeux and M. Masson. EVCLUS: Evidential Clustering of Proximity Data. IEEE Transactions on Systems, Man and Cybernetics B, 34(1):95-109, 2004.

[11] T. Denœux. Conjunctive and Disjunctive Combination of Belief Functions Induced by Non Distinct Bodies of Evidence. Artificial Intelligence, 172:234-264, 2008.

[12] T. Denœux. Extending stochastic ordering to belief functions on the real line. Information Sciences, 179(9):1362-1376, 2009.

[13] D. Dubois and R.R. Yager. Fuzzy set connectives as combinations of belief structures. Information Sciences, 66(3):245-276, 1992.

[14] D. Dubois, H. Prade, and Ph. Smets. Representing partial ignorance. IEEE Transactions on Systems, Man and Cybernetics, 26(3):361-377, 1996.

[15] M. El Najjar and P. Bonnifait. A road-matching method for precise vehicle localization using belief theory and Kalman filtering. Autonomous Robots, 19(2):173-191, 2005.

[16] D. Gruyer, C. Royère, R. Labayrade and D. Aubert. Credibilistic multi sensor fusion for real time application, application to obstacle detection and tracking. IEEE Int. Conf. on Advanced Robotics, ICAR'2003, paper P366, 2003.

[17] Y. Lemeret, E. Lefevre and D. Jolly. Improvement of an association algorithm for obstacle tracking. Information Fusion, 9(2):234-245, 2008. 
[18] D. Mercier, B. Quost and T. Denœux. Refined modeling of sensor reliability in the belief function framework using contextual discounting. Information Fusion, 9(2):246-258, 2008.

[19] D. Mercier, É. Lefèvre and D. Jolly. Object association in the TBM framework, application to vehicle driving aid. In the 6th International Symposium on Imprecise Probability: Theories and Applications, ISIPTA 2009, Durham, United Kingdom, July 14-18, 2009.

[20] D. Mercier, G. Cron, T. Denoeux and M.-H. Masson. Decision fusion for postal address recognition using belief functions. Expert Systems with Applications, 36(3):5643-5653, 2009.

[21] D. Mercier, T. Denœux and M.-H. Masson. Belief function correction mechanisms. Studies in Fuzziness and Soft Computing, B. BouchonMeunier et al. (Eds.), Vol. 249, pp. 203-222, January 2010.

[22] B. Ristic and Ph. Smets. Global cost of assignment in the TBM framework for association of uncertain ID reports. Aerospace Science and Technology, 11(4):303-309, 2007.

[23] M. Rombaut. Decision in multi-obstacle matching process using the theory of belief. Advances in Vehicle Control and Safety, AVCS98, pp. 63-68, 1998.

[24] M. Rombaut and V. Cherfaoui. Decision making in data fusion using Dempster-Shafer's theory. Symposium on Intelligent Components and Instrumentation for Control Applications, 1997.

[25] J. Schubert. Managing inconsistent intelligence. In Proceedings of the 3rd International Conference on Information Fusion, FUSION'2000, pp. TuB4/10-16, Paris, France, 2000.

[26] G. Shafer. A Mathematical Theory Of Evidence. Princeton University Press, Princeton, N.J., 1976.

[27] Ph. Smets. Belief functions: the disjunctive rule of combination and the generalized bayesian theorem. International Journal of Approximate Reasoning, 9:1-35, 1993.

[28] Ph. Smets. Decision making in the TBM: the necessity of the pignistic transformation. International Journal of Approximate Reasoning, 38(2):133-147, 2005. 
[29] Ph. Smets and R. Kennes. The transferable belief model. Artificial Intelligence, 66:191-243, 1994.

[30] Y.M. Wang and J.B. Yang and D.L. Xu and K.S. Chin. On the combination and normalization of interval-valued belief structures. Information Sciences, 177(5):1230-1247, 2007.

[31] W.Z. Wu. Attribute reduction based on evidence theory in incomplete decision systems. Information Sciences, 178(5):1355-1371, 2008. 